\title{
La Corruption : Un Probleme Socio-Economique Dans Les Euvres De Sony Labou Tansi
}

\author{
Nelson Chika Ogonwa \\ DOI: http://dx.doi.org/10.4314/ujah.v14i1.9
}

\section{Résumé}

L'indépendance des pays africain est censée apporter l'espoir et le contentement chez la masse populaire car elle marque la fin des régimes du pouvoir étranger. Malheureusement tous les bienfaits sont mieux imaginés. L'indépendance a provoqué une situation peu enviable dans la pluparts des pays africains, car leurs chefs d'états l'en a pris comme l'occasion d'acquérir la richesse alors que la vision d'évolution est à l'abandon. Avec cela, les écrivains africains de l'époque post indépendante sont passé de l'euphorie de la négritude à la critique de la mauvaise autorité qui existe dans le continent. Sony Labou Tansi était un des écrivains qui a utilisé ses ouvres littéraires à critiquer les mauvaises administrations surtout au Centre Afrique son pays natal. Ce travail, donc va mettre à nu vers une perspective sociocritique, les conséquences de la corruption dans les deux premiers romans (la vie et demie et l'état honteux) de Sony Labou Tansi.

\section{Abstract}

The independence of African countries was supposed to usher in a world of hope and fulfillment to the masses especially when it marked the end of a regime by foreign powers. Unfortunately, the benefits are better imagined. Independence brought about an unenviable status of most African countries as their new leaders saw it as an opportunity to amass wealth and abandon the vision of development. With this development, African writers of the post independent era shifted from the 
euphoria of negritude to a criticism of the practical realities of bad governance that reared up its ugly head in the continent. Sony Labou Tansi was one of the writers who used his literary works to attack the maladministration in place especially in the Central Africa where he comes from. This research work therefore, intends to expose through a socio-critical perspective, the effects of corruption as seen in the first two novels (la vie et demie and l'état honteux) of Sony Labou Tansi.

\section{L'Introduction}

La littérature africaine est marquée par les trois grandes périodes qui suivent: l'ère précoloniale, l'ère coloniale, et l'ère post coloniale. A l'époque postcoloniale, les œuvres littéraires africaines prennent l'homme Africaine comme sujet prédominant. Avec l'indépendance, on reconnait que les nouveaux états libres doivent déployer beaucoup d'effort pour se développer. Dans le domaine politique, on tend à éveiller une conscience nationale. Mais au contraire les moyens qu'emploient les dirigeants ne portent pas les fruits escomptés. On constate aussi la désillusion du peuple africaine qui aspire à une meilleure vie après l'indépendance. Par conséquent l'Afrique devient victime de plusieurs problèmes politiques comme la dictature et d'autres formes d'oppression et de la corruption.

Ayant observé ces problèmes survenus en Afrique d'après l'indépendance, les écrivains africains ne s'intéressent plus à l'euphorie de la négritude, mais ils préfèrent la vérité du témoignage sincère. Alors ils sont passés d'une critique dirigée contre une domination étrangère à une critique interne mettant en cause, certaines visions socio-économiques et politiques des nouvelles nations. 
Donc pour l'écrivain de l'Afrique post indépendante, sa plume devient sa seule arme de combat contre les maux et les dangers des régimes politiques en place. Parmi ces écrivains, on compte Sony Labou Tansi (écrivain congolais du $20^{\mathrm{e}}$ siècle). Tous les romans de celui-ci suivent de près les réalités africaines depuis La vie et demie(1979) jusqu' au Commencement des douleurs(1995) et L'Ante-peuple(1983).

Alors, notre travail de recherche tentera de répondre aux questions suivantes :

-Qu'est-ce que c'est la corruption et comment est-elle un problème socio-économique ?

-Le problème socio-économique est-il grave en Afrique indépendante?

-Comment peut on y remédier?

La réponse aux questions ci-dessus nous aide à faire saisir le conflit provoqué par la corruption en Afrique subsaharienne. Nous tenterons de répondre aux questions de la recherche par une analyse de la pensée littéraire de Sony Labou Tansi, c'est-à-dire la manière dont il a soulevé la corruption comme problème socio-économique dans la société congolaise en particulière et d'autres pays centre-africains en général. Pour ce faire, nous allons procéder à une analyse de la corruption dans ses deux premiers romans à savoir, La vie et demie(VD) et L'Etat honteux(EH). Nous faisons donc une analyse de la corruption dans le corpus de base dans la perspective de la sociocritique.

La sociocritique est une approche du fait littéraire qui s'attarde sur l'univers social dans le texte. C'est un mot crée par Claude Duchet en 1971, et elle propose une lecture sociohistorique du texte. La sociocritique nous permettra d'analyser la corruption comme pratique sociale que Tansi représente dans le corpus de base. Le pratique sociale entretient des rapports étroits avec sa société de référence. L' approche sociocritique nous permettra d'élucider les problèmes de la 
corruption dans le corpus, ainsi que les visions socioéconomiques de Tansi pour le développement de l'Afrique.

\section{La Corruption Comme Probleme Social En Afrique Independante}

La corruption est un fléau social qui se fait sentir dans beaucoup de romans africains. On en parle non seulement dans La vie et demie et L'Etat honteux de Sony Labou Tansi, mais aussi dans Xala de Sembene Ousmane,15 ans ca suffit! d'Amadou Ousmane et Les Chauves Souris de Bénard Nanga. A travers les œuvres ci-dessus, les auteurs essaient de dévoiler la maladresse des hommes politiques en Afrique, leur convoitise démesurée, qui les pousse à s'enrichir au détriment des paysans, ceux-ci étant condamnés à vivre plutôt dans la misère. Chez Tansi, on signale l'apogée du régime postcolonial à travers le Guide Providentiel et le Colonel Martillimi Lopez. Chez les deux dirigeants, on constate le détournement des biens de l'état. Ces personnages fabriquent des mensonges pour atteindre leur but égoïstes.

Dans La vie et demie, on condamne sans ménagement, la manipulation du budget national qui favorise la corruption. A l'aide d'un éloquent flashback, le docteur Tchi, ancien ministre chez le Guide Providentiel, nous présent ceci : «D'abord tu te fabriques des marches :médicalement, constructions, équipements ,missions. Un ministre est forme-tu dois savoir cette règle de jeu-un ministre est forme de vingt pour cent des dépenses de son ministère » (VD :34). Un ministre, une fois formé grâce à l'argent du public, gaspille l'argent de l'état au détriment du peuple. C'est une vie honteuse qui permet aux dirigeants de perpétuer la misère chez la masse populaire. Le narrateur soulève aussi le comportement des ministres corrompus à travers la vie ministérielle du Docteur Tchi : « Le docteur Tchi, comme on 
l'appelait à l'époque, mena la vie des VVVF Qu' on appelait la vie avec trois $\mathrm{V}$. Il construisit quatre villas, acheta une voiture a huit belles filles(...) Il vécut une vie vraiment ministérielle $»(\mathrm{VD}: 36)$. Autrement dit, le docteur Tchi se permet de gaspiller l'argent de l'Etat à travers des vins, des voitures, des villas et des femmes. C'est donc une occasion d'utiliser les finances publiques pour assouvir ses besoins personnels extravagants.

Dans L'Etat honteux, on constate aussi qu'il y a des gens qui dépensent l' argent du public dans des banques étrangères, issu de la corruption. Cela est présenté ainsi : «Il parlent de mon-colonel Carvanso chien couchante de sa hernie, ils parlent de son frère qui a foutu les finances nationales en Suisse comme si nous, on n'avait pas besoin d'argent $\gg(\mathrm{EH}: 42)$.

En plus, on soulève partout dans le texte que chaque régime autoritaire a un noyau de base qui constitue sa clef de voute. Ce sont justement les membres de ce cercle rapproché qui tirent le plus grand bénéfice du système. Tansi confirme cela dans L'Etat honteux quand il affirme par l'intermédiaire de Lopez que : «Et mon peuple je vais te dire : Tous ces mecs sauf moi-même Et Maman Nationale ont du fric en Suisse des milliards de coustrani qu'ils ont mis là-bas pour faire tourner l'Europe $\gg(\mathrm{EH}: 73)$.

Amadou Ousmane décrit aussi le même phénomène dans son œuvre 15 ans ca suffit! Là on nous indique comment le matérialisme grossier de certains privilégies tend à perpétuer la misère des plus démunis. Nous comprenons dans cette œuvre romanesque que Siddi Balima détourne les biens de l'Etat et s'enrichit de manière scandaleuse. Ce personnage se présente comme un politicien corrompu qui a des comtes bancaires à l'étranger ainsi que des biens mobiliers et immobiliers au Bentota. A travers son comportement et la mentalité d'autres dirigeants bentotais, l'auteur dénonce la politique de corruption qui ravage l'Afrique contemporaine. 
Parallèlement, la prostitution qui se manifeste un peu partout en Afrique contemporaine est issue des problèmes socioéconomiques. C'est la répression du Guide qui a provoqué la prostitution chez Chaidana. En justifiant ceci, Chaidana dit « je suis un produit de leur main :je les aurai tous $\gg(\mathrm{VD}: 46)$.

On rencontre aussi la prostitution chez le Guide-JeanCour-de-Pierre. On soulève ceci dans la semaine des vierges(VD :147). Là, le narrateur nous fait comprendre que le Guide a couché avec des vierges dont la scène a été radiodiffusée et télévisée dans la Katamalanasie(VD :147). Même chez le Colonel Lopez dans L'Etat honteux' on voit qu'il entretient un nombre insoutenable de maitresses, comme l'atteste sa parole qui s'adresse à Carvanso : «Puis il baissa un peu la voix pour lui demander si tu ne peut pas ah !Ma mère c'est dur la vie d'un célibataire:J'ai soif, cherche-moi un putain $\gg(\mathrm{EH}: 11)$.

Partout en Afrique, la corruption fait partie intégrante de la vie socio-économique. Elle apporte des résultats négatifs et c'est la masse populaire qui en subit l'influence.

\section{Les Consequences De La Corruption En Afrique Independante}

Tansi fait remarquer à travers notre corpus de base que le fonctionnement de l'indépendance du pays et de la politique de plusieurs nouveaux dirigeants noirs sont une négation de l'indépendance authentique. La plupart des dirigeants ne sont en effet qu' une clique d'arrivistes qui voient leurs positions comme une occasions pour s'enrichir au détriment de leurs concitoyens. Le Guide Providentiel et le Colonel Lopez possèdent des villas et des comptes bancaires à l'étranger. Ceci ne va pas sans entrainer aussi la pauvreté en Afrique. Parallèlement, le pouvoir d'achat de celle-ci devient de plus en 
plus faible. Au moyen de la corruption, la caisse nationale est vidée et la pauvreté s'installe.

Avec la corruption, les gouvernements que Tansi expose ne puissent pas améliorer la situation déplorable de l'économie. Dans L'Etat honteux, on remarque que Lopez n'a aucune solution aux problèmes économiques. Lorsqu'on lui demande d'exprimer son opinion vis-à-vis de la situation financière, il réplique : «Ah! Je me le disais, arrête, c'est une mauvaise question.....l'économie c'est une mixture préparée depuis la Flamandchourir, que voulez-vous que ma hernie y fasse ? »(EH : 142).

En général, la corruption entraine une crise dramatique au niveau de la vie économique. Quand les dirigeants ne peuvent pas améliorer la situation, elle peut provoquer la grève et une situation où les ouvriers protestent contre les mauvaises conditions du travail. Dans Les Bouts de Bois de Dieu de Sembene Ousmane, on signale que le salaire des travailleurs n'est plus compatible avec la réalité économique, et cette situation entraine irrésistiblement la grève.

En outre, la corruption provoque le détournement des biens publics. Les dirigeants profitent de ce vice social volent les biens de la nation et puis la caisse nationale est vidée ; alors que la population meurent dans la faim et dans la pauvreté. La gaspillage des bien publics chez le docteur Tchi dans la vie et demie et celui de Lopez et des membres de son cabinet dans l'état honteux entrainent la pauvreté dans leurs pays différents. Il faut signaler que la réalité économique dans notre corpus de base a des résonances actuelles dans les milieux géographiques qui constituent l'Afrique Centrale, y compris le pays natal de Tansi, le Congo-Brazzaville. Lylian Kesteloot résume cette situation deshumanisante dans cette région d'une manière succincte: «On vit les répressions, les corruptions, les détournements des richesses des Etats au profit de ceux qui étaient chargés de les gérer pour le bien public »(Kesteloot, $2001: 252$ ). 
Avec la situation économique déplorable en katamalanasie et celle de l'Afrique Centrale, on peut dire que certains pays africains ne sont pas indépendants sur le plan économique.

\section{La Vision Socio-Economique De Tansi}

La vision socio-économique du romancier se voit dans son rejet de toute forme d'oppression qui se trouve dans la politique de l'Afrique indépendante.

Parlant de la vie économique, on constate comment Tansi présente un devoir sacré en conseillant à l'Afrique de rejeter catégoriquement l'approche désastreuse de plusieurs dirigeants Africains qui n'ont aucune solutions aux problèmes économiques. Par exemple, l'incapacité de Lopez (dans L'Etat honteux) de proposer des solutions vis-à-vis de la situation financière traduit bien la nature honteuse de son règne. Alors pour Tansi, ces dirigeants font partie des corbeaux et vautours qui survolent l'Afrique indépendante.

Tansi dénonce aussi sans ambages le nouveau "prédateur', c'est-à-dire les agents de la violence fait prendre conscience de la situation déplorable de la vie économique dans laquelle le négro-africain se trouve et il faut relever le défi.

Chez la femme, Tansi utilise des traits négatifs pour effectuer la chute du régime du Guide Providentiel. Le romancier dépeint, par exemple, la prostitution pour avoir un changement positif dans la vie politique de la Katamalanasie. Là, on voit des femmes qui se servent de leur beauté ensorcelante pour triompher des despotes. Les deux Chaidana, la mère comme la fille usent de leur beauté comme une arme destructive contre le despotisme du Guide et ses successeurs.

Comme moyen de résoudre les problèmes, Chaidana (la mère) utilise des armes sexuelles pour éliminer l'un après 
l'autre presque tous les dignitaires du gouvernement du Guide afin de stopper le despotisme en place. On constate qu'en faisant l'amour, Chaidana rend son «sang pourri »aux dignitaires du pays(VD :48)Ce pouvoir de séduction, croyonsnous, est une arme symbolique que Tansi à donné aux personnages féminins.

Il faut noter qu'en représentant les activités des deux Chaidana, Tansi n'encourage pas pour autant la prostitution. Mais il se sert de leur charme de beauté pour influencer des régimes politiques afin d'apporter des changements positifs dans la société. A travers les rôles que les deux Chaidana ont joués dans l'œuvre, on soulève le féminisme chez Tansi. Les Chaidana revendiquent la promotion et la défense des droits des femmes, tout en invitant la masse populaire opprimée à prendre conscience de sa situation actuelle. A travers toutes ces manifestations, Tansi estime que les femmes sont indispensables à apporter des changements positifs dans des situations déplorables.

Enfin, Tansi prêche la révolution en vue d'apporter des changements significatifs au niveau de la vie économique des pays transposés dans le corpus retenu.

\section{Conclusion}

Dans notre corpus de base, Tansi se préoccupe des contradictions économiques dans la société congolaise et d'autres pays centre- africains d'après les indépendances africaines. Les deux textes de Tansi servent d'instrument de contestation et d'éveil de conscience chez la masse populaire. Tansi utilise les deux antihéros- le Guide Providentiel et le Colonel Martillimi Lopez- pour critiquer certains dirigeants africains qui ne font que détruire l'économie de leurs domines à travers la corruption. En bref, ce sont des leaders politiques qui sont responsables de la misère qui ravage leurs différentes régions géographiques en Afrique. 
Pour une amélioration significative du système politique, il faut bien de l'honnêteté de la part des dirigeants et des citoyens. Les femmes se doivent également de se réveiller et de lutter contre les régimes qui ne s'intéressent qu'à perpétuer la misère chez le peuple. Et pour la masse populaire, il ne faut pas être faible et nonchalant devant le problème de la corruption.

Nelson Chika Ogonwa is of the Department of Languages, Delta State Polytechnic, Ogwashi-Uku

\section{Cuvres Citees}

Alain, Rouche et Gerald Clavreuil. Littérature Nationales d'Ecriture Française. Histoire Littéraire et Anthologie. Paris : Bordas, 1987.

Atangana, Nicholas. Problématique du développement en Afrique Tropicale. Yaoundé : Edition CLE, 1978.

Bamidele, L.O. Literature and sociology. Ibadan: StirlingHorden Publishers ltd. 2000.

Chemain, Roger. L'imaginaire dans le roman africain. Paris : L'Harmattan, 1986.

Fantoure, Alioum. Le cercle des tropiques. Paris: Présence Africaine, 1972.

Kesteloot, Lylian. Histoire de le littérature négro-africaine. Paris: Edition Karthala,2001.

Korouma, Ahmadou. Les soleils des indépendances. Paris : Edition du seuil, 1970.

Ousmane, Amadou. 15 ans ca suffit! Dakar: Les Nouvelles Editions Africaine, 1985.

Ousmane, Sembene. Les Bouts de bois de Dieu. Paris : Presse Pocket, 1960.

Tansi, Sony Labou. La vie et demie. Paris: Edition du Seuil, 1979 
Tansi, Sony Labou. L'Etat honteux. Paris : Edition du Seuil, 1981.

Seuil, 1983.

L'Ante- peuple. Paris: Edition du 